\title{
Accelerated Lyra's Cosmology Driven by Electromagnetic Field in Inhomogeneous Universe
}

\author{
Anirudh Pradhan ${ }^{1}$ and Padmini Yadav ${ }^{2}$ \\ ${ }^{1}$ Department of Mathematics, Hindu P. G. College, Zamania, Ghazipur, Uttar Pardesh 232 331, India \\ ${ }^{2}$ Department of Mathematics, P. G. College, Ghazipur 233 001, India
}

Correspondence should be addressed to Anirudh Pradhan, acpradhan@yahoo.com

Received 13 July 2009; Accepted 11 December 2009

Recommended by Christian Corda

\begin{abstract}
A new class of cylindrically symmetric inhomogeneous cosmological models for perfect fluid distribution with electromagnetic field is obtained in the context of Lyra's geometry. We have obtained solutions by considering the time dependent displacement field. The source of the magnetic field is due to an electric current produced along the $z$-axis. Only $F_{12}$ is a nonvanishing component of electromagnetic field tensor. To get the deterministic solution, it has been assumed that the expansion $\theta$ in the model is proportional to the shear $\sigma$. It has been found that the solutions are consistent with the recent observations of type Ia supernovae, and the displacement vector $\beta(t)$ affects entropy. Physical and geometric aspects of the models are also discussed in presence and absence of magnetic field.
\end{abstract}

Copyright (c) 2009 A. Pradhan and P. Yadav. This is an open access article distributed under the Creative Commons Attribution License, which permits unrestricted use, distribution, and reproduction in any medium, provided the original work is properly cited.

\section{Introduction and Motivations}

The inhomogeneous cosmological models play a significant role in understanding some essential features of the universe such as the formation of galaxies during the early stages of evolution and process of homogenization. The early attempts at the construction of such models have been done by Tolman [1] and Bondi [2] who considered spherically symmetric models. Inhomogeneous plane-symmetric models were considered by Taub $[3,4]$ and later by Tomimura [5], Szekeres [6], Collins and Szafron [7, 8], and Szafron and Collins [9]. Senovilla [10] obtained a new class of exact solutions of Einstein's equations without big bang singularity, representing a cylindrically symmetric, inhomogeneous cosmological model filled with perfect fluid which is smooth and regular everywhere satisfying energy and causality conditions. Later, Ruiz and Senovilla [11] have examined a fairly large class of singularity-free models through a comprehensive study of general cylindrically symmetric metric with separable function of $r$ and $t$ as metric coefficients. 
Dadhich et al. [12] have established a link between the FRW model and the singularityfree family by deducing the latter through a natural and simple in-homogenization and anisotropization of the former. Recently, Patel et al. [13] have presented a general class of inhomogeneous cosmological models filled with nonthermalized perfect fluid assuming that the background space-time admits two space-like commuting Killing vectors and has separable metric coefficients. Singh et al. [14] obtained inhomogeneous cosmological models of perfect fluid distribution with electromagnetic field. Recently, Pradhan et al. [15-18] have investigated cylindrically-symmetric inhomogeneous cosmological models in various contexts.

The occurrence of magnetic field on galactic scale is a well-established fact today, and its importance for a variety of astrophysical phenomena is generally acknowledged as pointed out by Zeldovich et al. [19]. Also Harrison [20] suggests that magnetic field could have a cosmological origin. As natural consequences, we should include magnetic fields in the energy-momentum tensor of the early universe. The choice of anisotropic cosmological models in Einstein system of field equations leads to the cosmological models more general than Robertson-Walker model [21]. The presence of primordial magnetic field in the early stages of the evolution of the universe is discussed by many [22-31]. Strong magnetic field can be created due to adiabatic compression in clusters of galaxies. Large-scale magnetic field gives rise to anisotropies in the universe. The anisotropic pressure created by the magnetic fields dominates the evolution of the shear anisotropy and decays slowly as compared to the case when the pressure is held isotropic $[32,33]$. Such fields can be generated at the end of an inflationary epoch [34-38]. Anisotropic magnetic field models have significant contribution in the evolution of galaxies and stellar objects. Bali and Ali [39] obtained a magnetized cylindrically symmetric universe with an electrically neutral perfect fluid as the source of matter. Pradhan et al. [40-44] have investigated magnetized cosmological models in various contexts.

In 1917 Einstein introduced the cosmological constant into his field equations of general relativity in order to obtain a static cosmological model since, as is well known, without the cosmological term his field equations admit only nonstatic solutions. After the discovery of the red-shift of galaxies and explanation thereof Einstein regretted for the introduction of the cosmological constant. Recently, there has been much interest in the cosmological term in context of quantum field theories, quantum gravity, super-gravity theories, Kaluza-Klein theories and the inflationary-universe scenario. Shortly after Einstein's general theory of relativity Weyl [45] suggested the first so-called unified field theory based on a generalization of Riemannian geometry. With its backdrop, it would seem more appropriate to call Weyl's theory a geometrized theory of gravitation and electromagnetism (just as the general theory was a geometrized theory of gravitation only), instead of a unified field theory. It is not clear as to what extent the two fields have been unified, even though they acquire (different) geometrical significance in the same geometry. The theory was never taken seriously inasmuchas it was based on the concept of nonintegrability of length transfer; and, as pointed out by Einstein, this implies that spectral frequencies of atoms depend on their past histories and therefore have no absolute significance. Nevertheless, Weyl's geometry provides an interesting example of nonRiemannian connections, and recently Folland [46] has given a global formulation of Weyl manifolds clarifying considerably many of Weyl's basic ideas thereby.

In 1951 Lyra [47] proposed a modification of Riemannian geometry by introducing a gauge function into the structureless manifold, as a result of which the cosmological constant arises naturally from the geometry. This bears a remarkable resemblance to Weyl's 
geometry. But in Lyra's geometry, unlike that of Weyl, the connection is metric preserving as in Remannian; in other words, length transfers are integrable. Lyra also introduced the notion of a gauge and in the "normal" gauge the curvature scalar in identical to that of Weyl. In consecutive investigations Sen [48], Sen and Dunn [49] proposed a new scalar-tensor theory of gravitation and constructed an analog of the Einstein field equations based on Lyra's geometry. It is, thus, possible [48] to construct a geometrized theory of gravitation and electromagnetism much along the lines of Weyl's "unified" field theory, however, without the inconvenience of nonintegrability length transfer.

Halford [50] has pointed out that the constant vector displacement field $\phi_{i}$ in Lyra's geometry plays the role of cosmological constant $\Lambda$ in the normal general relativistic treatment. It is shown by Halford [51] that the scalar-tensor treatment based on Lyra's geometry predicts the same effects within observational limits as the Einstein's theory. Several authors Sen and Vanstone [52], Bhamra [53], Karade and Borikar [54], Kalyanshetti and Waghmode [55], Reddy and Innaiah [56], Beesham [57], Reddy and Venkateswarlu [58], Soleng [59], have studied cosmological models based on Lyra's manifold with a constant displacement field vector. However, this restriction of the displacement field to be constant is merely one for convenience, and there is no a priori reason for it. Beesham [60] considered FRW models with time-dependent displacement field. He has shown that by assuming the energy density of the universe to be equal to its critical value, the models have the $k=-1$ geometry. T. Singh and G. P. Singh [61-64], Singh and Desikan [65] have studied Bianchi-type I, III, Kantowaski-Sachs and a new class of cosmological models with time-dependent displacement field and have made a comparative study of Robertson-Walker models with constant deceleration parameter in Einstein's theory with cosmological term and in the cosmological theory based on Lyra's geometry. Soleng [59] has pointed out that the cosmologies based on Lyra's manifold with constant gauge vector $\phi$ will either include a creation field and be equal to Hoyle's creation field cosmology [66-68] or contain a special vacuum field, which together with the gauge vector term, may be considered as a cosmological term. In the latter case the solutions are equal to the general relativistic cosmologies with a cosmological term.

Recently, Pradhan et al. [69-73], Casana et al. [74], Rahaman et al. [75, 76], Bali and Chandnani [77], Kumar and Singh [78], Singh [79], Rao, Vinutha et al. [80] and Pradhan [81] have studied cosmological models based on Lyra's geometry in various contexts. Rahaman et al. [82, 83] have evaluated solutions for plane-symmetric thick domain wall in Lyra geometry by using the separable form for the metric coefficients. Rahaman [84-87] has also studied some topological defects within the framework of Lyra geometry. With these motivations, in this paper, we have obtained exact solutions of Einstein's modified field equations in cylindrically symmetric inhomogeneous space-time within the frame work of Lyra's geometry in the presence and absence of magnetic field for time varying displacement vector. This paper is organized as follows. In Section 1 the motivation for the present work is discussed. The metric and the field equations are presented in Section 2. In Section 3 the solutions of field equations are derived for time varying displacement field $\beta(t)$ in presence of magnetic field. Section 4 contains the physical and geometric properties of the model in presence of magnetic field. The solutions in absence of magnetic field are given in Section 5. The physical and geometric properties of the model in absence of magnetic field are discussed in Section 6. Finally, in Section 7 discussion and concluding remarks are given. 


\section{The Metric and Field Equations}

We consider the cylindrically symmetric metric in the form

$$
d s^{2}=A^{2}\left(d x^{2}-d t^{2}\right)+B^{2} d y^{2}+C^{2} d z^{2}
$$

where $A$ is the function of $t$, alone and $B$ and $C$ are functions of $x$ and $t$. The energy momentum tensor is taken as having the form

$$
T_{i}^{j}=(\rho+p) u_{i} u^{j}+p g_{i}^{j}+E_{i}^{j},
$$

where $\rho$ and $p$ are, respectively, the energy density and pressure of the cosmic fluid, and $u_{i}$ is the fluid four-velocity vector satisfying the condition

$$
u^{i} u_{i}=-1, \quad u^{i} x_{i}=0
$$

In (2.2), $E_{i}^{j}$ is the electromagnetic field given by Lichnerowicz [88]

$$
E_{i}^{j}=\bar{\mu}\left[h_{l} h^{l}\left(u_{i} u^{j}+\frac{1}{2} g_{i}^{j}\right)-h_{i} h^{j}\right]
$$

where $\bar{\mu}$ is the magnetic permeability and $h_{i}$ the magnetic flux vector defined by

$$
h_{i}=\frac{1}{\bar{\mu}}{ }^{*} F_{j i} u^{j}
$$

where the dual electromagnetic field tensor ${ }^{*} F_{i j}$ is defined by Synge [89]

$$
{ }^{*} F_{i j}=\frac{\sqrt{-g}}{2} \epsilon_{i j k l} F^{k l}
$$

Here $F_{i j}$ is the electromagnetic field tensor and $\epsilon_{i j k l}$ is the Levi-Civita tensor density.

The coordinates are considered to be comoving so that $u^{1}=0=u^{2}=u^{3}$ and $u^{4}=$ $1 / A$. If we consider that the current flows along the $z$-axis, then $F_{12}$ is the only nonvanishing component of $F_{i j}$. The Maxwell's equations

$$
\begin{aligned}
& F[i j ; k]=0, \\
& {\left[\frac{1}{\bar{\mu}} F^{i j}\right]_{; j}=0,}
\end{aligned}
$$

require that $F_{12}$ is the function of $x$-alone. We assume that the magnetic permeability is the functions of $x$ and $t$ both. Here the semicolon represents a covariant differentiation. 
The field equations (in gravitational units $c=1, G=1$ ), in normal gauge for Lyra's manifold, were obtained by Sen [48] as

$$
R_{i j}-\frac{1}{2} g_{i j} R+\frac{3}{2} \phi_{i} \phi_{j}-\frac{3}{4} g_{i j} \phi_{k} \phi^{k}=-8 \pi T_{i j}
$$

where $\phi_{i}$ is the displacement field vector defined as

$$
\phi_{i}=(0,0,0, \beta(t)),
$$

where other symbols have their usual meaning as in Riemannian geometry.

For the line-element (2.1), the field (2.8) with (2.2) and (2.9) leads to the following system of equations:

$$
\begin{gathered}
\frac{1}{A^{2}}\left[-\frac{\ddot{B}}{B}-\frac{\ddot{C}}{C}+\frac{\dot{A}}{A}\left(\frac{\dot{B}}{B}+\frac{\dot{C}}{C}\right)-\frac{\dot{B} \dot{C}}{B C}+\frac{B^{\prime} C^{\prime}}{B C}\right]-\frac{3}{4} \beta^{2}=8 \pi\left(p+\frac{F_{12}^{2}}{2 \bar{\mu} A^{2} B^{2}}\right), \\
\frac{1}{A^{2}}\left(\frac{\dot{A}^{2}}{A^{2}}-\frac{\ddot{A}}{A}-\frac{\ddot{C}}{C}+\frac{C^{\prime \prime}}{C}\right)-\frac{3}{4} \beta^{2}=8 \pi\left(p+\frac{F_{12}^{2}}{2 \bar{\mu} A^{2} B^{2}}\right) \\
\frac{1}{A^{2}}\left(\frac{\dot{A}^{2}}{A^{2}}-\frac{\ddot{A}}{A}-\frac{\ddot{B}}{B}+\frac{B^{\prime \prime}}{B}\right)-\frac{3}{4} \beta^{2}=8 \pi\left(p-\frac{F_{12}^{2}}{2 \bar{\mu} A^{2} B^{2}}\right), \\
\frac{1}{A^{2}}\left[-\frac{B^{\prime \prime}}{B}-\frac{C^{\prime \prime}}{C}+\frac{\dot{A}}{A}\left(\frac{\dot{B}}{B}+\frac{\dot{C}}{C}\right)-\frac{B^{\prime} C^{\prime}}{B C}+\frac{\dot{B} \dot{C}}{B C}\right]+\frac{3}{4} \beta^{2}=8 \pi\left(\rho+\frac{F_{12}^{2}}{2 \bar{\mu} A^{2} B^{2}}\right), \\
\frac{\dot{B}^{\prime}}{B}+\frac{\dot{C}^{\prime}}{C}-\frac{\dot{A}}{A}\left(\frac{B^{\prime}}{B}+\frac{C^{\prime}}{C}\right)=0 .
\end{gathered}
$$

Here, and also in the following expressions, a dot and a dash indicate ordinary differentiation with, respect to $t$ and $x$ respectively.

The energy conservation equation $T_{i ; j}^{i}=0$ leads to

$$
\begin{gathered}
\dot{\rho}+(\rho+p)\left(\frac{2 \dot{A}}{A}+\frac{\dot{B}}{B}+\frac{\dot{C}}{C}\right)=0 \\
\left(R_{i}^{j}-\frac{1}{2} g_{i}^{j} R\right)_{; j}+\frac{3}{2}\left(\phi_{i} \phi^{j}\right)_{; j}-\frac{3}{4}\left(g_{i}^{j} \phi_{k} \phi^{k}\right)_{; j}=0 .
\end{gathered}
$$

Equation (2.16) leads to

$$
\frac{3}{2} \phi_{i}\left[\frac{\partial \phi^{j}}{\partial x^{j}}+\phi^{l} \Gamma_{l j}^{j}\right]+\frac{3}{2} \phi^{j}\left[\frac{\partial \phi_{i}}{\partial x^{j}}-\phi_{l} \Gamma_{i j}^{l}\right]-\frac{3}{4} g_{i}^{j} \phi_{k}\left[\frac{\partial \phi^{k}}{\partial x^{j}}+\phi^{l} \Gamma_{l j}^{k}\right]-\frac{3}{4} g_{i}^{j} \phi^{k}\left[\frac{\partial \phi_{k}}{\partial x^{j}}+\phi_{l} \Gamma_{k j}^{l}\right]=0 .
$$


Equation (2.17) is identically satisfied for $i=1,2,3$. For $i=4$, (2.17) reduces to

$$
\frac{3}{2} \beta \dot{\beta}+\frac{3}{2} \beta^{2}\left(\frac{2 \dot{A}}{A}+\frac{\dot{B}}{B}+\frac{\dot{C}}{C}\right)=0
$$

\section{Solution of Field Equations in Presence of Magnetic Field}

Equations (2.10)-(2.14) are five independent equations in seven unknowns $A, B, C, \rho, p, \beta$, and $F_{12}$. For the complete determinacy of the system, we need two extra conditions which are narrated hereinafter. The research on exact solutions is based on some physically reasonable restrictions used to simplify the field equations.

To get determinate solution, we assume that the expansion $\theta$ in the model is proportional to the shear $\sigma$. This condition leads to

$$
A=\left(\frac{B}{C}\right)^{n}
$$

where $n$ is a constant. The motive behind assuming this condition is explained with reference to Thorne [90]; the observations of the velocity-red-shift relation for extragalactic sources suggest that Hubble expansion of the universe is isotropic today within $\approx 30$ percent $[91,92]$. To put more precisely, red-shift studies place the limit

$$
\frac{\sigma}{H} \leq 0.3
$$

on the ratio of shear, $\sigma$, to Hubble constant, $H$, in the neighbourhood of our Galaxy today. Collins et al. [93] have pointed out that for spatially homogeneous metric, the normal congruence to the homogeneous expansion satisfies that the condition $\sigma / \theta$ is constant.

From (2.15)-(2.17), we have

$$
\begin{gathered}
\frac{A_{44}}{A}-\frac{A_{4}^{2}}{A^{2}}+\frac{A_{4} B_{4}}{A B}+\frac{A_{4} C_{4}}{A C}-\frac{B_{44}}{B}-\frac{B_{4} C_{4}}{B C}=\frac{C_{11}}{C}-\frac{B_{1} C_{1}}{B C}=\mathrm{K} \text { (constant) } \\
\frac{8 \pi F_{12}^{2}}{\bar{\mu} B^{2}}=-\frac{C_{44}}{C}+\frac{C_{11}}{C}+\frac{B_{44}}{B}-\frac{B_{11}}{B} .
\end{gathered}
$$

We also assume that

$$
\begin{aligned}
& B=f(x) g(t), \\
& C=f(x) k(t) .
\end{aligned}
$$


Using (3.1) and (3.5) in (2.14) and (3.3) leads to

$$
\begin{gathered}
\frac{k_{4}}{k}=\frac{(2 n-1)}{(2 n+1)} \frac{g_{4}}{g}, \\
(n-1) \frac{g_{44}}{g}-n \frac{k_{44}}{k}-\frac{g_{4}}{g} \frac{k_{4}}{k}=K, \\
f f_{11}-f_{1}^{2}=K f^{2} .
\end{gathered}
$$

Equation (3.6) leads to

$$
k=c_{0} g^{\alpha},
$$

where $\alpha=(2 n-1) /(2 n+1)$ and $c_{0}$ is the constant of integration. From (3.7) and (3.9), we have

$$
\frac{g_{44}}{g}+\ell \frac{g_{4}^{2}}{g^{2}}=N
$$

where

$$
\ell=\frac{n \alpha(\alpha-1)+\alpha}{n(\alpha-1)+1}, \quad N=\frac{K}{n(1-\alpha)-1} .
$$

Equation (3.8) leads to

$$
f=\exp \left(\frac{1}{2} K\left(x+x_{0}\right)^{2}\right)
$$

where $x_{0}$ is an integrating constant. Equation (3.10) leads to

$$
g=\left(c_{1} e^{b t}+c_{2} e^{-b t}\right)^{1 /(\ell+1)}
$$

where $b=\sqrt{(\ell+1) N}$ and $c_{1}, c_{2}$ are integrating constants. Hence from (3.9) and (3.13), we have

$$
k=c_{0}\left(c_{1} e^{b t}+c_{2} e^{-b t}\right)^{\alpha /(\ell+1)}
$$


Therefore, we obtain

$$
\begin{gathered}
B=\exp \left(\frac{1}{2} K\left(x+x_{0}\right)^{2}\right)\left(c_{1} e^{b t}+c_{2} e^{-b t}\right)^{1 /(\ell+1)} \\
C=\exp \left(\frac{1}{2} K\left(x+x_{0}\right)^{2}\right) c_{0}\left(c_{1} e^{b t}+c_{2} e^{-b t}\right)^{\alpha /(\ell+1)} \\
A=a\left(c_{1} e^{b t}+c_{2} e^{-b t}\right)^{n(1-\alpha) /(\ell+1)}
\end{gathered}
$$

where $a=c_{3} / c_{0}, c_{3}$ being a constant of integration.

After using suitable transformation of the coordinates, the model (2.1) reduces to the form

$$
\begin{aligned}
d s^{2}= & a^{2}\left(c_{1} e^{b T}+c_{2} e^{-b T}\right)^{2 n(1-\alpha) /(\ell+1)}\left(d X^{2}-d T^{2}\right) \\
& +e^{K X^{2}}\left(c_{1} e^{b T}+c_{2} e^{-b T}\right)^{2 /(\ell+1)} d Y^{2}+e^{K X^{2}}\left(c_{1} e^{b T}+c_{2} e^{-b T}\right)^{2 \alpha /(\ell+1)} d Z^{2}
\end{aligned}
$$

where $x+x_{0}=X, t=T, y=Y$, and $c_{0} z=Z$.

\section{Some Physical and Geometric Properties of the Model in Presence of Magnetic Field}

Equation (2.18) gives

$$
\frac{\dot{\beta}}{\beta}=-\left(\frac{2 \dot{A}}{A}+\frac{\dot{B}}{B}+\frac{\dot{C}}{C}\right), \quad \text { as } \beta \neq 0,
$$

which leads to

$$
\frac{\dot{\beta}}{\beta}=-\frac{b\{2 n(1-\alpha)+1+\alpha\}}{(\ell+1)}\left[\frac{c_{1} e^{b T}-c_{2} e^{-b T}}{c_{1} e^{b T}+c_{2} e^{-b T}}\right] .
$$

Equation (4.2) on integration gives

$$
\beta=K_{0}\left(c_{1} e^{b T}+c_{2} e^{-b T}\right)^{\kappa}
$$

where $K_{0}$ is a constant of integration and

$$
\mathcal{\kappa}=\frac{b\{2 n(\alpha-1)-(\alpha+1)\}}{(\ell+1)} .
$$


Using (3.15) and (4.3) in (2.10) and (2.13), the expressions for pressure $p$ and density $\rho$ for the model (3.16) are given by

$$
\begin{aligned}
8 \pi p= & \frac{1}{a^{2} \psi_{2}^{2 n(1-\alpha) /(\ell+1)}}\left[K^{2} X^{2}-\frac{2(3+\alpha) b^{2} c_{1} c_{2}}{(\ell+1) \psi_{2}^{2}}-\frac{\left(2 n \alpha^{2}+\alpha^{2}+2 \alpha-2 n+3\right) b^{2}}{2(\ell+1)^{2}} \frac{\psi_{1}^{2}}{\psi_{2}^{2}}\right]-\frac{3}{4} K_{0}^{2} \psi_{2}^{2 \kappa}, \\
8 \pi \rho= & \frac{1}{a^{2} \psi_{2}^{2 n(1-\alpha) /(\ell+1)}}\left[-3 K^{2} X^{2}-2 K+\frac{2 b^{2}(\alpha-1) c_{1} c_{2}}{(\ell+1) \psi_{2}^{2}}-\frac{\left(2 n \alpha^{2}-\alpha^{2}-2 \alpha-2 n+1\right) b^{2}}{2(\ell+1)^{2}} \frac{\psi_{1}^{2}}{\psi_{2}^{2}}\right] \\
& +\frac{3}{4} K_{0}^{2} \psi_{2}^{2 \kappa},
\end{aligned}
$$

where

$$
\begin{aligned}
& \psi_{1}=c_{1} e^{b T}-c_{2} e^{-b T} \\
& \psi_{2}=c_{1} e^{b T}+c_{2} e^{-b T} .
\end{aligned}
$$

From (3.4) the nonvanishing component $F_{12}$ of the electromagnetic field tensor is obtained as

$$
F_{12}^{2}=\frac{\bar{\mu}}{8 \pi} \frac{b^{2}(1-\alpha)}{(\ell+1)^{2}} e^{K X^{2}} \psi_{2}^{2 /(\ell+1)}\left[\frac{4(\ell+1) c_{1} c_{2}+(1+\alpha) \psi_{1}^{2}}{\psi_{2}^{2}}\right] .
$$

From the above, equation it is observed that the electromagnetic field tensor increases with time.

The reality conditions (Ellis [94])

$$
\begin{aligned}
& \text { (i) } \rho+p>0, \\
& \text { (ii) } \rho+3 p>0 \text {, }
\end{aligned}
$$

lead to

$$
\begin{gathered}
\frac{b^{2}\left(n-n \alpha^{2}-1\right)}{(\ell+1)^{2}} \frac{\psi_{1}^{2}}{\psi_{2}^{2}}-\frac{4 b^{2} c_{1} c_{2}}{(\ell+1) \psi_{2}^{2}}>K\left(K X^{2}+1\right), \\
\frac{b^{2}\left(4 n-4 n \alpha^{2}-\alpha^{2}-2 \alpha-5\right)}{(\ell+1)^{2}} \frac{\psi_{1}^{2}}{\psi_{2}^{2}}-\frac{4 b^{2}(\alpha+5) c_{1} c_{2}}{(\ell+1) \psi_{2}^{2}}>2 K+\frac{3}{2} \beta^{2} a^{2} \psi_{2}^{2 n(1-\alpha) /(\ell+1)},
\end{gathered}
$$

respectively.

The dominant energy conditions (Hawking and Ellis [95])
(i) $\rho-p \geq 0$,
(ii) $\rho+p \geq 0$, 
lead to

$$
\begin{gathered}
\frac{b^{2}(\alpha+1)^{2}}{(\ell+1)^{2}} \frac{\psi_{1}^{2}}{\psi_{2}^{2}}+\frac{4 b^{2}(\alpha+1) c_{1} c_{2}}{(\ell+1) \psi_{2}^{2}}+\frac{3}{2} \beta^{2} a^{2} \psi_{2}^{2 n(1-\alpha) /(\ell+1)} \geq 2 K\left(2 K X^{2}+1\right), \\
\frac{b^{2}\left(n-n \alpha^{2}-1\right)}{(\ell+1)^{2}} \frac{\psi_{1}^{2}}{\psi_{2}^{2}}-\frac{4 b^{2} c_{1} c_{2}}{(\ell+1) \psi_{2}^{2}} \geq K\left(K X^{2}+1\right),
\end{gathered}
$$

respectively. The conditions (4.10) and (4.12) impose a restriction on displacement vector $\beta(t)$.

The expressions for the expansion $\theta$, Hubble parameter $H$, shear scalar $\sigma^{2}$, deceleration parameter $q$, and proper volume $V^{3}$ for the model (3.16) are given by

$$
\begin{gathered}
H=3 \theta=\frac{3 b\{n(1-\alpha)+(1+\alpha)\}}{(\ell+1) a \psi_{2}^{n(1-\alpha) /(\ell+1)}} \frac{\psi_{1}}{\psi_{2}}, \\
\sigma^{2}=\frac{b^{2}\left[\{n(1-\alpha)+(1+\alpha)\}^{2}-3 n(1-\alpha)(1+\alpha)-3 \alpha\right]}{3(\ell+1)^{2} a^{2} \psi_{2}^{2 n(1-\alpha) /(\ell+1)}} \frac{\psi_{1}^{2}}{\psi_{2}^{2}} \\
q=-1-\frac{6 c_{1} c_{2}(\ell+1)}{n\left(1-\alpha^{2}\right) \psi_{1}^{2}}, \\
V^{3}=\sqrt{-g}=a^{2} \psi_{2}^{2 n(1+\alpha)(1-\alpha) /(\ell+1)} e^{K X^{2}} .
\end{gathered}
$$

From (4.14) and (4.15), we obtain

$$
\frac{\sigma^{2}}{\theta^{2}}=\frac{\{n(1-\alpha)+(1+\alpha)\}^{2}-3 n\left(1-\alpha^{2}\right)-3 \alpha}{3\{n(1-\alpha)+(1+\alpha)\}^{2}}=\text { constant. }
$$

The rotation $\omega$ is identically zero.

The rate of expansion $H_{i}$ in the direction of $x, y$, and $z$ is given by

$$
\begin{gathered}
H_{x}=\frac{A_{4}}{A}=\frac{n b(1-\alpha)}{(\ell+1)} \frac{\psi_{1}}{\psi_{2}} \\
H_{y}=\frac{B_{4}}{B}=\frac{b}{(\ell+1)} \frac{\psi_{1}}{\psi_{2}} \\
H_{z}=\frac{C_{4}}{C}=\frac{b \alpha}{(\ell+1)} \frac{\psi_{1}}{\psi_{2}} .
\end{gathered}
$$

Generally the model (3.16) represents an expanding, shearing, and nonrotating universe in which the flow vector is geodetic. The model (3.16) starts expanding at $T>0$ and goes on expanding indefinitely when $n(1-\alpha) /(\ell+1)<0$. Since $\sigma / \theta=$ constant, the model does not approach isotropy. As $T$ increases the proper volume also increases. The physical quantities $p$ and $\rho$ decrease as $F_{12}$ increases. However, if $n(1-\alpha) /(\beta+1)>0$, the process of contraction 
starts at $T>0$, and at $T=\infty$ the expansion stops. The electromagnetic field tensor does not vanish when $b \neq 0$, and $\alpha \neq 1$. It is observed from (4.16) that $q<0$ when $c_{1}>0$ and $c_{2}>0$ which implies an accelerating model of the universe. Recent observations of type Ia supernovae [96-100] reveal that the present universe is in accelerating phase and deceleration parameter lies somewhere in the range $-1<q \leq 0$. It follows that our models of the universe are consistent with recent observations. Either when $c_{1}=0$ or $c_{2}=0$, the deceleration parameter $q$ approaches the value $(-1)$ as in the case of de-Sitter universe.

\section{Field Equations and Their Solution in Absence of Magnetic Field}

In absence of magnetic field, the field (2.8) with (2.2) and (2.9) for metric (2.1) reads as

$$
\begin{gathered}
\frac{1}{A^{2}}\left[-\frac{B_{44}}{B}-\frac{C_{44}}{C}+\frac{A_{4}}{A}\left(\frac{B_{4}}{B}+\frac{C_{4}}{C}\right)-\frac{B_{4} C_{4}}{B C}+\frac{B_{1} C_{1}}{B C}\right]=8 \pi p+\frac{3}{4} \beta^{2}, \\
\frac{1}{A^{2}}\left(\frac{A_{4}^{2}}{A^{2}}-\frac{A_{44}}{A}-\frac{C_{44}}{C}+\frac{C_{11}}{C}\right)=8 \pi p+\frac{3}{4} \beta^{2}, \\
\frac{1}{A^{2}}\left(\frac{A_{4}^{2}}{A^{2}}-\frac{A_{44}}{A}-\frac{B_{44}}{B}+\frac{B_{11}}{B}\right)=8 \pi p+\frac{3}{4} \beta^{2}, \\
\frac{1}{A^{2}}\left[-\frac{B_{11}}{B}-\frac{C_{11}}{C}+\frac{A_{4}}{A}\left(\frac{B_{4}}{B}+\frac{C_{4}}{C}\right)-\frac{B_{1} C_{1}}{B C}+\frac{B_{4} C_{4}}{B C}\right]=8 \pi \rho-\frac{3}{4} \beta^{2}, \\
\frac{B_{14}}{B}+\frac{C_{14}}{C}-\frac{A_{4}}{A}\left(\frac{B_{1}}{B}+\frac{C_{1}}{C}\right)=0 .
\end{gathered}
$$

Equations (5.2) and (5.3) lead to

$$
\frac{B_{44}}{B}-\frac{B_{11}}{B}-\frac{C_{44}}{C}+\frac{C_{11}}{C}=0 .
$$

Equations (3.5) and (5.6) lead to

$$
\frac{g_{44}}{g}-\frac{k_{44}}{k}=0 .
$$

Equations (3.9) and (5.7) lead to

$$
\frac{g_{44}}{g}+\alpha \frac{g_{4}^{2}}{g^{2}}=0
$$

which on integration gives

$$
g=\left(c_{4} t+c_{5}\right)^{1 /(\alpha+1)},
$$


where $c_{4}$ and $c_{5}$ are constants of integration. Hence from (3.9) and (5.9), we have

$$
k=c_{0}\left(c_{4} t+c_{5}\right)^{\alpha /(\alpha+1)} .
$$

In this case (3.8) also leads to the same as (3.12).

Therefore, in absence of magnetic field, we have

$$
\begin{gathered}
B=\exp \left(\frac{1}{2} K\left(x+x_{0}\right)^{2}\right)\left(c_{4} t+c_{5}\right)^{1 /(\alpha+1)}, \\
C=\exp \left(\frac{1}{2} K\left(x+x_{0}\right)^{2}\right) c\left(c_{4} t+c_{5}\right)^{\alpha /(\alpha+1)}, \\
A=a\left(c_{4} t+c_{5}\right)^{n(1-\alpha) /(1+\alpha)},
\end{gathered}
$$

where $a$ is already defined in previous section.

After using suitable transformation of the coordinates, the metric (2.1) reduces to the form

$$
d s^{2}=a^{2}\left(c_{4} T\right)^{2 n(1-\alpha) /(1+\alpha)}\left(d X^{2}-d T^{2}\right)+e^{K X^{2}}\left(c_{4} T\right)^{2 /(\alpha+1)} d Y^{2}+e^{K X^{2}}\left(c_{4} T\right)^{2 \alpha /(\alpha+1)} d Z^{2},
$$

where $x+x_{0}=X, y=Y, c_{0} z=Z$, and $t+c_{5} / c_{4}=T$.

\section{Some Physical and Geometric Properties of the Model in Absence of Magnetic Field}

With the use of (5.11), equation (2.18) leads to

$$
\frac{\dot{\beta}}{\beta}=\frac{1}{T}\left[\frac{2 n(\alpha-1)-(\alpha+1)}{(\alpha+1)}\right], \quad \text { as } \beta \neq 0,
$$

which upon integration leads to

$$
\beta=\Re T^{((2 n(\alpha-1)-(\alpha+1)) /(\alpha+1))},
$$

where $\mathfrak{R}$ is an integrating constant.

Using (5.11) and (6.2) in (5.1) and (5.4), the expressions for pressure $p$ and density $\rho$ for the model (5.12) are given by

$$
\begin{gathered}
8 \pi p=\frac{1}{a^{2}\left(c_{4} T\right)^{2 n(1-\alpha) /(1+\alpha)}}\left[\left\{\frac{n\left(1-\alpha^{2}\right)+\alpha}{(\alpha+1)^{2}}\right\} \frac{1}{T^{2}}+K^{2} X^{2}\right]-\frac{3}{4} \mathfrak{R}^{2} T^{2((2 n(\alpha-1)-(\alpha+1)) /(\alpha+1)),} \\
8 \pi \rho=\frac{1}{a^{2}\left(c_{4} T\right)^{2 n(1-\alpha) /(1+\alpha)}}\left[\left\{\frac{n\left(1-\alpha^{2}\right)+\alpha}{(\alpha+1)^{2}}\right\} \frac{1}{T^{2}}-K\left(2+3 K X^{2}\right)\right]+\frac{3}{4} \mathfrak{R}^{2} T^{2((2 n(\alpha-1)-(\alpha+1)) /(\alpha+1))} .
\end{gathered}
$$


International Journal of Mathematics and Mathematical Sciences

The dominant energy conditions (Hawking and Ellis [95])

$$
\begin{aligned}
& \text { (i) } \quad \rho-p \geq 0 \text {, } \\
& \text { (ii) } \quad \rho+p \geq 0 \text {, }
\end{aligned}
$$

lead to

$$
\begin{gathered}
\frac{3}{4} \beta^{2} a^{2}\left(c_{4} T\right)^{2 n(1-\alpha) /(1+\alpha)} \geq K\left(1+2 K X^{2}\right) \\
\left\{\frac{n\left(1-\alpha^{2}\right)+\alpha}{(1+\alpha)^{2}}\right\} \frac{1}{T^{2}} \geq K\left(1+K X^{2}\right)
\end{gathered}
$$

respectively.

The reality conditions (Ellis [94])

$$
\begin{aligned}
& \text { (i) } \rho+p>0, \\
& \text { (ii) } \rho+3 p>0 \text {, }
\end{aligned}
$$

lead to

$$
\begin{gathered}
\left\{\frac{n\left(1-\alpha^{2}\right)+\alpha}{(1+\alpha)^{2}}\right\} \frac{1}{T^{2}}>K\left(1+K X^{2}\right), \\
\frac{2\left[n\left(1-\alpha^{2}\right)+\alpha\right]}{(1+\alpha)^{2}} \frac{1}{T^{2}}>K+\frac{3}{4} \beta^{2}\left(c_{4} T\right)^{2 n(1-\alpha) /(1+\alpha)} .
\end{gathered}
$$

The conditions (6.5) and (6.9) impose a restriction on $\beta(t)$.

The expressions for the expansion $\theta$, Hubble parameter $H$, shear scalar $\sigma^{2}$, deceleration parameter $q$, and proper volume $V^{3}$ for the model (5.12) in absence of magnetic field are given by

$$
\begin{gathered}
H=3 \theta=\frac{n(1-\alpha)+(1+\alpha)}{a(1+\alpha) c_{4}^{n(1-\alpha) /(1+\alpha)}} \frac{1}{T^{(n(1-\alpha)+(1+\alpha)) /(1+\alpha)}}, \\
\sigma^{2}=\frac{\{n(1-\alpha)+(1+\alpha)\}^{2}-3 n\left(1-\alpha^{2}\right)-3 \alpha}{3 a^{2}(1+\alpha)^{2} c_{4}^{n(1-\alpha) /(1+\alpha)}} \frac{1}{T^{(2 n(1-\alpha)+2(1+\alpha)) /(1+\alpha)}}, \\
q=-1+\frac{3(\alpha+1)}{2 n(1-\alpha)+2(1+\alpha)}, \\
V^{3}=\sqrt{-g}=a^{2} e^{K X^{2}}\left(c_{4} T\right)^{(2 n(1-\alpha)+(1+\alpha)) /(1+\alpha)} .
\end{gathered}
$$


From (6.10) and (6.11), we obtain

$$
\frac{\sigma^{2}}{\theta^{2}}=\frac{\{n(1-\alpha)+(1+\alpha)\}^{2}-3 n\left(1-\alpha^{2}\right)-3 \alpha}{3\{n(1-\alpha)+(1+\alpha)\}^{2}}=\text { constant. }
$$

The rotation $\omega$ is identically zero.

The rate of expansion $H_{i}$ in the direction of $x, y$, and $z$ are given by

$$
\begin{aligned}
& H_{x}=\frac{A_{4}}{A}=\frac{n(1-\alpha)}{(1+\alpha)} \frac{1}{T} \\
& H_{y}=\frac{B_{4}}{B}=\frac{1}{(1+\alpha)} \frac{1}{T}, \\
& H_{z}=\frac{C_{4}}{C}=\frac{\alpha}{(1+\alpha)} \frac{1}{T} .
\end{aligned}
$$

The model (5.12) starts expanding with a big bang at $T=0$ and it stops expanding at $T=\infty$. It should be noted that the universe exhibits initial singularity of the Point-type at $T=0$. The space-time is well behaved in the range $0<T<T_{0}$. In absence of magnetic field, the model represents a shearing and nonrotating universe in which the flow vector is geodetic. At the initial moment $T=0$, the parameters $\rho, p, \beta, \theta, \sigma^{2}$ and $H$ tend to infinity. So the universe starts from initial singularity with infinite energy density, infinite internal pressure, infinitely large gauge function, infinite rate of shear and expansion. Moreover, $\rho, p, \beta, \theta, \sigma^{2}$ and $H$ are monotonically decreasing toward a nonzero finite quantity for $T$ in the range $0<T<T_{0}$ in absence of magnetic field. Since $\sigma / \theta=$ constant, the model does not approach isotropy. As $T$ increases the proper volume also increases. It is observed that for the derived model, the displacement vector $\beta(t)$ is a decreasing function of time and therefore it behaves like cosmological term $\Lambda$. It is observed from (6.12) that $q<0$ when $\alpha<(2 n-1) /(2 n+1)$ which implies an accelerating model of the universe. When $\alpha=-1$, the deceleration parameter $q$ approaches the value $(-1)$ as in the case of de-Sitter universe. Thus, also in absence of magnetic field, our models of the universe are consistent with recent observations.

\section{Discussion and Concluding Remarks}

In this paper, we have obtained a new class of exact solutions of Einstein's modified field equations for cylindrically symmetric space-time with perfect fluid distribution within the framework of Lyra's geometry both in presence and absence of magnetic field. The solutions are obtained using the functional separability of the metric coefficients. The source of the magnetic field is due to an electric current produced along the $z$-axis. $F_{12}$ is the only nonvanishing component of electromagnetic field tensor. The electromagnetic field tensor is given by (4.7), $\bar{\mu}$ remains undetermined as function of both $x$ and $t$. The electromagnetic field tensor does not vanish if $b \neq 0$ and $\alpha \neq 1$. It is observed that in presence of magnetic field, the rate of expansion of the universe is faster than that in absence of magnetic field. The idea of primordial magnetism is appealing because it can potentially explain all the large-scale fields seen in the universe today, specially those found in remote proto-galaxies. As a result, the literature contains many studies examining the role and the implications of magnetic 
fields for cosmology. In presence of magnetic field, the model (3.16) represents an expanding, shearing and nonrotating universe in which the flow vector is geodetic. But in the absence of magnetic field, the model (5.12) found that in the universe all the matter and radiation are concentrated at the big bang epoch and the cosmic expansion is driven by the big bang impulse. The universe has singular origin and it exhibits power-law expansion after the big bang impulse. The rate of expansion slows down and finally stops at $T \rightarrow \infty$. In absence of magnetic field, the pressure, energy density and displacement field become zero whereas the spatial volume becomes infinitely large as $T \rightarrow \infty$.

It is possible to discuss entropy in our universe. In thermodynamics the expression for entropy is given by

$$
T d S=d\left(\rho V^{3}\right)+p\left(d V^{3}\right)
$$

where $V^{3}=A^{2} B C$ is the proper volume in our case. To solve the entropy problem of the standard model, it is necessary to treat $d S>0$ for at least a part of evolution of the universe. Hence (7.1) reduces to

$$
T d S=\rho_{4}+(\rho+p)\left(2 \frac{A_{4}}{A}+\frac{B_{4}}{B}+\frac{C_{4}}{C}\right)>0 .
$$

The conservation equation $T_{i: j}^{j}=0$ for (2.1) leads to

$$
\rho_{4}+(\rho+p)\left(\frac{A_{4}}{A}+\frac{B_{4}}{B}+\frac{C_{4}}{C}\right)+\frac{3}{2} \beta \beta_{4}+\frac{3}{2} \beta^{2}\left(2 \frac{A_{4}}{A}+\frac{B_{4}}{B}+\frac{C_{4}}{C}\right)=0 .
$$

Therefore, (7.1) and (7.2) lead to

$$
\frac{3}{2} \beta \beta_{4}+\frac{3}{2} \beta^{2}\left(2 \frac{A_{4}}{A}+\frac{B_{4}}{B}+\frac{C_{4}}{C}\right)<0,
$$

which gives to $\beta<0$. Thus, the displacement vector $\beta(t)$ affects entropy because for entropy $d S>0$ leads to $\beta(t)<0$.

In spite of homogeneity at large scale, our universe is inhomogeneous at small scale, so physical quantities being position-dependent are more natural in our observable universe if we do not go to super high scale. This result shows this kind of physical importance. It is observed that the displacement vector $\beta(t)$ coincides with the nature of the cosmological constant $\Lambda$ which has been supported by the work of several authors as discussed in the physical behaviour of the model in Sections 4 and 6 . In the recent time $\Lambda$-term has attracted theoreticians and observers for many a reason. The nontrivial role of the vacuum in the early universe generates a $\Lambda$-term that leads to inflationary phase. Observationally, this term provides an additional parameter to accommodate conflicting data on the values of the Hubble constant, the deceleration parameter, the density parameter and the age of the universe (see, e.g., $[101,102]$ ). In recent past there is an upsurge of interest in scalar fields in general relativity and alternative theories of gravitation in the context of inflationary cosmology [103-105]. Therefore the study of cosmological models in Lyra's geometry may be 
relevant for inflationary models. There seems a good possibility of Lyra's geometry to provide a theoretical foundation for relativistic gravitation, astrophysics, and cosmology. However, the importance of Lyra's geometry for astrophysical bodies is still an open question. In fact, it needs a fair trial for experiment.

\section{Acknowledgments}

The authors would like to thank the Harish-Chandra Research Institute, Allahabad, India for local hospitality where this work is done. The authors also thank the referee for his fruitful comments.

\section{References}

[1] R. C. Tolman, "Effect of inhomogeneity on cosmological models," Proceedings of the National Academy of Sciences, vol. 20, no. 3, pp. 169-176, 1934.

[2] H. Bondi, "Spherically symmetrical models in general relativity," Royal Astronomical Society, vol. 107, pp. 410-425, 1947.

[3] A. H. Taub, "Empty space-times admitting a three parameter group of motions," Annals of Mathematics, vol. 53, pp. 472-490, 1951.

[4] A. H. Taub, "Isentropic hydrodynamics in plane symmetric space-times," Physical Review, vol. 103, pp. $454-467,1956$.

[5] N. Tomimura, "Evolution of inhomogeneous plane-symmetric cosmological models," Il Nuovo Cimento B Series, vol. 44, no. 2, pp. 372-380, 1978.

[6] P. Szekeres, "A class of inhomogeneous cosmological models," Communications in Mathematical Physics, vol. 41, pp. 55-64, 1975.

[7] C. B. Collins and D. A. Szafron, "A new approach to inhomogeneous cosmologies: instinsic symmetries I," Journal of Mathematical Physics, vol. 20, pp. 2347-2353, 1979.

[8] C. B. Collins and D. A. Szafron, "A new approach to inhomogeneous cosmologies: instinsic symmetries III," Journal of Mathematical Physics, vol. 20, pp. 2362-2370, 1979.

[9] D. A. Szafron and C. B. Collins, "A new approach to inhomogeneous cosmologies: intrinsic symmetries. II. Conformally flat slices and an invariant classification," Journal of Mathematical Physics, vol. 20, no. 11, pp. 2354-2361, 1979.

[10] J. M. M. Senovilla, "New class of inhomogeneous cosmological perfect-fluid solutions without bigbang singularity," Physical Review Letters, vol. 64, no. 19, pp. 2219-2221, 1990.

[11] E. Ruiz and J. M. M. Senovilla, "General class of inhomogeneous perfect-fluid solutions," Physical Review D, vol. 45, no. 6, pp. 1995-2005, 1992.

[12] N. Dadhich, R. Tikekar, and L. K. Patel, "On singularity free cosmological models," Current Science, vol. 65, p. 694, 1993.

[13] L. K. Patel, R. Tikekar, and N. Dadhich, "Inhomogeneous cosmological models with heat flux," Pramana-Journal of Physics, vol. 49, no. 2, pp. 213-224, 1997.

[14] G. Singh, P. Mehta, and S. Gupta, "Some inhomogeneous perfect fluid cosmological models in the presence of electromagnetic field," Astrophysics and Space Science, vol. 281, no. 4, pp. 677-688, 2002.

[15] A. Pradhan, P. K. Singh, and R. Kanti Jotania, "Cylindrically symmetric inhomogeneous cosmological models with viscous fluid and varying $\Lambda, "$ Czechoslovak Journal of Physics, vol. 56, no. 6, pp. 641-660, 2006.

[16] A. Pradhan, A. Rai, and S. K. Singh, "Cylindrically symmetric inhomogeneous universe with electromagnetic field in string cosmology," Astrophysics and Space Science, vol. 312, no. 3-4, pp. 261265, 2007.

[17] A. Pradhan, "Magnetized string cosmological model in cylindrically-symmetric inhomogeneous universe with variable cosmological term $\Lambda$," Fizika B, vol. 16, no. 4, pp. 205-222, 2007.

[18] A. Pradhan, K. Jotania, and A. Singh, "Magnetized string cosmological model in cylindrically symmetric inhomogeneous universe with time dependent cosmological-term $\Lambda$," Brazilian Journal of Physics, vol. 38, no. 1, pp. 167-177, 2008. 
[19] Ya. B. Zeldovich, A. A. Ruzmainkin, and D. D. Sokoloff, Magnetic Field in Astrophysics, Gordon and Breach, New York, NY, USA, 1993.

[20] E. R. Harrison, "Origin of magnetic fields in the early universe," Physical Review Letters, vol. 30, no. 5, pp. 188-190, 1973.

[21] A. G. Walker, "On Milne's theory of world-structure," Proceedings of the London Mathematical Society, vol. 42, no. 1, pp. 90-127, 1937.

[22] C. W. Misner, K. S. Thorne, and J. A. Wheeler, Gravitation, W. H. Freeman, San Francisco, Calif, USA, 1973.

[23] E. Asseo and H. Sol, “Extragalatic magnetic fields," Physics Reports, vol. 148, no. 6, pp. 307-435, 1987.

[24] M. A. Melvin, "Homogeneous axial cosmologies with electromagnetic fields and dust ${ }^{\mathrm{fn} 1}$," Annals of the New York Academy of Sciences, vol. 262, pp. 253-274, 1975.

[25] R. E. Pudritz and J. Silk, "The origin of magnetic fields and primordial stars in protogalaxies," Astrophysical Journal, vol. 342, pp. 650-659, 1989.

[26] K.-T. Kim, P. P. Kronberg, and P. C. Tribble, "Detection of excess rotation measure due to intracluster magnetic fields in clusters of galaxies," Astrophysical Journal, vol. 379, no. 1, pp. 80-88, 1991.

[27] R. A. Perley and G. B. Taylor, "VLA observations of 3C 295-a young radio galaxy?" Astronomical Journal, vol. 101, no. 5, pp. 1623-1631, 1991.

[28] P. P. Kronberg, J. J. Perry, and E. L. H. Zukowski, “Discovery of extended faraday rotation compatible with spiral structure in an intervening galaxy at $z=0.395$ : new observations of PKS 1229-021," Astrophysical Journal, vol. 387, no. 2, pp. 528-535, 1992.

[29] A. M. Wolfe, K. M. Lanzetta, and A. L. Oren, "Magnetic fields in damped Ly-alpha systems," Astrophysical Journal, vol. 388, no. 1, pp. 17-22, 1992.

[30] R. M. Kulsrud, R. Cen, J. P. Ostriker, and D. Ryu, "The protogalactic origin for cosmic magnetic fields," Astrophysical Journal, vol. 480, no. 2, part 1, pp. 481-491, 1997.

[31] E. G. Zweibel and C. Heiles, "Magnetic fields in galaxies and beyond," Nature, vol. 385, no. 6612, pp. 131-136, 1997.

[32] J. D. Barrow, "Cosmological limits on slightly skew stresses," Physical Review D, vol. 55, no. 12, pp. 7451-7460, 1997.

[33] Ya. B. Zeldovich, Soviet Astronomy, vol. 13, p. 608, 1970.

[34] M. S. Turner and L. M. Widrow, "Inflation-produced, large-scale magnetic fields," Physical Review D, vol. 37, no. 10, pp. 2743-2754, 1988.

[35] J. M. Quashnock, A. Loeb, and D. N. Spergel, "Magnetic field generation during the cosmological QCD phase transition," Astrophysical Journal, vol. 344, pp. L49-L51, 1989.

[36] B. Ratra, "Cosmological "seed" magnetic field from inflation," Astrophysical Journal, vol. 391, no. 1, part 2, pp. L1-L4, 1992.

[37] A. Dolgov and J. Silk, "Electric charge asymmetry of the Universe and magnetic field generation," Physical Review D, vol. 47, no. 8, pp. 3144-3150, 1993.

[38] A. D. Dolgov, "Breaking of conformal invariance and electromagnetic field generation in the Universe," Physical Review D, vol. 48, no. 6, pp. 2499-2501, 1993.

[39] R. Bali and M. Ali, "Magnetized cylindrically symmetric universe in general relativity," PramanaJournal of Physics, vol. 47, no. 1, pp. 25-31, 1996.

[40] A. Pradhan and O. P. Pandey, "Bianchi type I anisotropic magnetized cosmological models with varying $\Lambda$, " International Journal of Modern Physics D, vol. 12, no. 7, pp. 1299-1314, 2003.

[41] A. Pradhan, S. K. Srivastav, and K. R. Jotania, "Some inhomogeneous magnetized viscous-fluid cosmological models with varying $\Lambda, "$ Czechoslovak Journal of Physics, vol. 54, no. 2, pp. 255-272, 2004.

[42] A. Pradhan and S. K. Singh, "Bianchi type I magnetofluid cosmological models with variable cosmological constant revisited," International Journal of Modern Physics D, vol. 13, no. 3, pp. 503516, 2004.

[43] A. Pradhan, P. Pandey, and K. K. Rai, "Magnetized anisotropic cosmological models with varying $\Lambda$, , Czechoslovak Journal of Physics, vol. 56, no. 3, pp. 303-313, 2006.

[44] A. Pradhan, A. K. Yadav, and J. P. Singh, "A new class of plane-symmetric inhomogeneous cosmological models of perfect fluid distribution with electromagnetic field," Fizika B, vol. 16, pp. 175-196, 2007.

[45] H. Weyl, Sber. Preussische Akademie der Wissenschaften zu Berlin, p. 465, 1918.

[46] G. B. Folland, "Weyl manifolds," Journal of Differential Geometry, vol. 4, pp. 145-153, 1970.

[47] G. Lyra, "Über eine Modifikation der Riemannschen Geometrie," Mathematische Zeitschrift, vol. 54, pp. 52-64, 1951. 
[48] D. K. Sen, “A static cosmological model," Zeitschrift für Physik C, vol. 149, pp. 311-323, 1957.

[49] D. K. Sen and K. A. Dunn, "A scalar-tensor theory of gravitation in a modified Riemannian manifold," Journal of Mathematical Physics, vol. 12, pp. 578-586, 1971.

[50] W. D. Halford, "Cosmological theory based on Lyra's geometry," Australian Journal of Physics, vol. 23, p. 863, 1970.

[51] W. D. Halford, "Scalar-tensor theory of gravitation in a Lyra manifold," Journal of Mathematical Physics, vol. 13, no. 11, pp. 1699-1703, 1972.

[52] D. K. Sen and J. R. Vanstone, "On Weyl and Lyra manifolds," Journal of Mathematical Physics, vol. 13, pp. 990-993, 1972.

[53] K. S. Bhamra, "A cosmological model of class one in Lyra's manifold," Australian Journal of Physics, vol. 27, pp. 541-547, 1974.

[54] T. M. Karade and S. M. Borikar, "Thermodynamic equilibrium of a gravitating sphere in Lyra's geometry," General Relativity and Gravitation, vol. 9, no. 5, pp. 431-436, 1978.

[55] S. B. Kalyanshetti and B. B. Waghmode, "A static cosmological model in Einstein-Cartan theory," General Relativity and Gravitation, vol. 14, no. 10, pp. 823-830, 1982.

[56] D. R. K. Reddy and P. Innaiah, "A plane symmetric cosmological model in Lyra manifold," Astrophysics and Space Science, vol. 123, no. 1, pp. 49-52, 1986.

[57] A. Beesham, "Friedmann's cosmology in Lyra's manifold," Astrophysics and Space Science, vol. 127, no. 2, pp. 355-359, 1986.

[58] D. R. K. Reddy and R. Venkateswarlu, "Birkhoff-type theorem in the scale-covariant theory of gravitation," Astrophysics and Space Science, vol. 136, no. 1, pp. 191-194, 1987.

[59] H. H. Soleng, "Cosmologies based on Lyra's geometry," General Relativity and Gravitation, vol. 19, no. 12, pp. 1213-1216, 1987.

[60] A. Beesham, "FLRW cosmological models in Lyra's manifold with time dependent displacement field," Australian Journal of Physics, vol. 41, no. 6, pp. 833-842, 1988.

[61] T. Singh and G. P. Singh, "Bianchi type-I cosmological models in Lyra's geometry," Journal of Mathematical Physics, vol. 32, no. 9, pp. 2456-2458, 1991.

[62] T. Singh and G. P. Singh, "Some cosmological models with constant deceleration parameter," Il Nuovo Cimento B, vol. 106, no. 6, pp. 617-622, 1991.

[63] T. Singh and G. P. Singh, "Bianchi type III and Kantowski-Sachs cosmological models in Lyra geometry," International Journal of Theoretical Physics, vol. 31, no. 8, pp. 1433-1446, 1992.

[64] T. Singh and G. P. Singh, "Lyra's geometry and cosmology: a review," Fortschritte der Physik, vol. 41, no. 8, pp. 737-764, 1993.

[65] G. P. Singh and K. Desikan, "A new class of cosmological models in Lyra geometry," PramanaJournal of Physics, vol. 49, no. 2, pp. 205-212, 1997.

[66] F. Hoyle, Monthly Notices of the Royal Astronomical Society, vol. 108, p. 252, 1948.

[67] F. Hoyle and J. V. Narlikar, "Mach's principle and the creation of matter," Proceedings of the Royal Society A, vol. 273, pp. 1-11, 1963.

[68] F. Hoyle and J. V. Narlikar, "A new theory of gravitation," Proceedings of the Royal Society A, vol. 282, pp. 191-207, 1964.

[69] A. Pradhan, I. Aotemshi, and G. P. Singh, "Plane symmetric domain wall in Lyra geometry," Astrophysics and Space Science, vol. 288, no. 3, pp. 315-325, 2003.

[70] A. Pradhan and A. K. Vishwakarma, "A new class of LRS Bianchi type-I cosmological models in Lyra geometry," Journal of Geometry and Physics, vol. 49, no. 3-4, pp. 332-342, 2004.

[71] A. Pradhan, L. Yadav, and A. K. Yadav, "Isotropic homogeneous universe with a bulk viscous fluid in Lyra geometry," Astrophysics and Space Science, vol. 299, no. 1, pp. 31-42, 2005.

[72] A. Pradhan, V. Rai, and S. Otarod, "Plane symmetric bulk viscous domain wall in Lyra geometry," Fizika B, vol. 15, no. 2, pp. 57-70, 2006.

[73] A. Pradhan, K. K. Rai, and A. K. Yadav, "Plane symmetric bulk viscous domain wall in lyra geometry," Brazilian Journal of Physics, vol. 37, no. 3B, pp. 1084-1093, 2007.

[74] R. Casana, C. A. M. De Melo, and B. M. Pimentel, "Spinorial field and Lyra geometry," Astrophysics and Space Science, vol. 305, no. 2, pp. 125-132, 2006.

[75] F. Rahaman, B. C. Bhui, and G. Bag, "Can Lyra geometry explain the singularity free as well as accelerating universe?" Astrophysics and Space Science, vol. 295, no. 4, pp. 507-513, 2005.

[76] F. Rahaman, S. Das, N. Begum, and M. Hossain, Astrophysics and Space Science, vol. 295, p. 507, 2005.

[77] R. Bali and N. K. Chandnani, "Bianchi type-I cosmological model for perfect fluid distribution in Lyra geometry," Journal of Mathematical Physics, vol. 49, no. 3, Article ID 032502, 8 pages, 2008. 
[78] S. Kumar and C. P. Singh, "An exact Bianchi type-I cosmological model in Lyra's manifold," International Journal of Modern Physics A, vol. 23, no. 6, pp. 813-822, 2008.

[79] J. K. Singh, "Exact solutions of some cosmological models in Lyra geometry," Astrophysics and Space Science, vol. 314, no. 4, pp. 361-365, 2008.

[80] V. U. M. Rao, T. Vinutha, and M. V. Santhi, "Bianchi type-V cosmological model with perfect fluid using negative constant deceleration parameter in a scalar tensor theory based on Lyra manifold," Astrophysics and Space Science, vol. 314, no. 1-3, pp. 213-216, 2008.

[81] A. Pradhan, "Cylindrically symmetric viscous fluid universe in Lyra geometry," Journal of Mathematical Physics, vol. 50, no. 2, Article ID 022501, 13 pages, 2009.

[82] F. Rahaman, S. Chakraborty, and M. Kalam, "Domain wall in Lyra geometry," International Journal of Modern Physics D, vol. 10, no. 5, pp. 735-739, 2001.

[83] F. Rahaman, M. Kalam, and R. Mondal, "Thin domain walls in Lyra Geometry," Astrophysics and Space Science, vol. 305, no. 4, pp. 337-340, 2006.

[84] F. Rahaman, "Global monopoles in Lyra geometry," International Journal of Modern Physics D, vol. 9, no. 6, pp. 775-779, 2000.

[85] F. Rahaman, S. Chakraborty, and J. K. Bera, "A study of an inhomogeneous Bianchi-I model in Lyra geometry," Astrophysics and Space Science, vol. 281, no. 3, pp. 595-600, 2002.

[86] F. Rahaman, N. Begum, and S. Das, "A class of higher dimensional spherically symmetric cosmological model in Lyra geometry," Astrophysics and Space Science, vol. 294, no. 3-4, pp. 219-224, 2004.

[87] F. Rahaman and P. Ghosh, "General relativistic domain wall in Lyra geometry," Fizika B, vol. 13, no. 4, pp. 719-724, 2004.

[88] A. Lichnerowicz, Relativistic Hydrodynamics and Magnetohydrodynamics, W. A. Benzamin, New York, NY, USA, 1967.

[89] J. L. Synge, Relativity: The General Theory, Series in Physics, North-Holland, Amsterdam, The Netherlands, 1960.

[90] K. S. Thorne, "Primordial element formation, primordial magnetic fields, and the isotropy of the universe," The Astrophysical Journal, vol. 148, p. 51, 1967.

[91] R. Kantowski and R. K. Sachs, "Some spatially homogeneous anisotropic relativistic cosmological models," Journal of Mathematical Physics, vol. 7, no. 3, pp. 443-446, 1966.

[92] J. Kristian and R. K. Sachs, "Observations in cosmology," The Astrophysical Journal, vol. 143, pp. 379399, 1966.

[93] C. B. Collins, E. N. Glass, and D. A. Wilkinson, "Exact spatially homogeneous cosmologies," General Relativity and Gravitation, vol. 12, no. 10, pp. 805-823, 1980.

[94] G. F. R. Ellis, in General Relativity and Cosmology, R. K. Sachs, Ed., p. 117, Clarendon Press, Oxford, UK, 1973.

[95] S. W. Hawking and G. F. R. Ellis, The Large Scale Structure of Space-Time, Cambridge Monographs on Mathematical Physics, Cambridge University Press, London, UK, 1973.

[96] S. Perlmutter, S. Gabi, G. Goldhaber, et al., "Measurements1 of the cosmological parameters $\Omega$ and $\Lambda$ from the first seven supernovae at $z \geq 0.35$," Astrophysical Journal, vol. 483, no. 2, part 1, pp. 565-581, 1997.

[97] S. Perlmutter, G. Aldering, M. Della Valle, et al., "Discovery of a supernova explosion at half the age of the universe," Nature, vol. 391, no. 6662, pp. 51-54, 1998.

[98] S. Perlmutter, G. Aldering, G. Goldhaber, et al., "Measurements of $\Omega$ and $\Lambda$ from 42 high-redshift Supernovae," Astrophysical Journal, vol. 517, no. 2, part 1, pp. 565-586, 1999.

[99] A. G. Riess, A. V. Filippenko, P. Challis, et al., "Observational evidence from supernovae for an accelerating universe and a cosmological constant," Astronomical Journal, vol. 116, no. 3, pp. 1009$1038,1998$.

[100] A. G. Riess, L.-G. Sirolger, J. Tonry, et al., "Type Ia supernova discoveries at $z>1$ from the hubble space telescope: evidence for past deceleration and constraints on dark energy evolution," Astrophysical Journal, vol. 607, no. 2, part 1, pp. 665-687, 2004.

[101] J. E. Gunn and B. M. Tinsley, "An accelerating universe," Nature, vol. 257, no. 5526, pp. 454-457, 1975.

[102] E. J. Wampler and W. L. Burke, in New Ideas in Astronomy, F. Bertola, J. W. Sulentix, and B. F. Madora, Eds., p. 317, Cambridge University Press, Cambridge, UK, 1988.

[103] G. F. R. Ellis, Standard and Inflationary Cosmologies, SISSA, Trieste, Italy, 1990. 
[104] D. La and P. J. Steinhardt, "Extended inflationary cosmology," Physical Review Letters, vol. 62, no. 4, pp. 376-378, 1989.

[105] J. D. Barrow, “Graduated inflationary universes,” Physics Letters B, vol. 235, no. 1-2, pp. 40-43, 1990. 


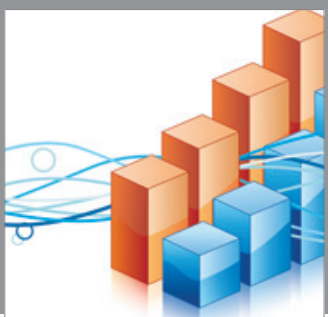

Advances in

Operations Research

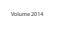

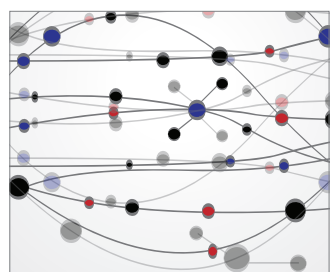

\section{The Scientific} World Journal
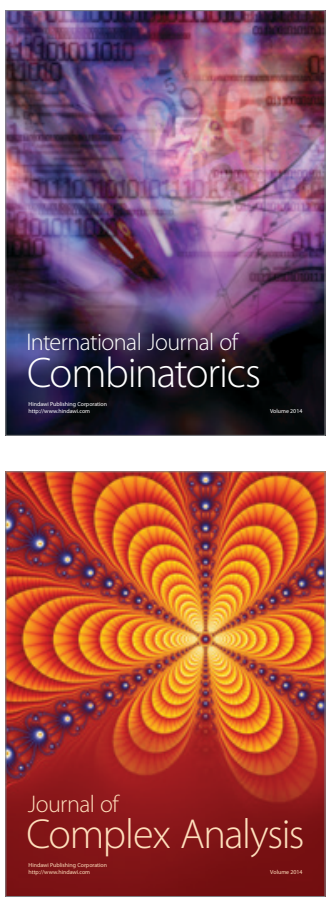

International Journal of

Mathematics and

Mathematical

Sciences
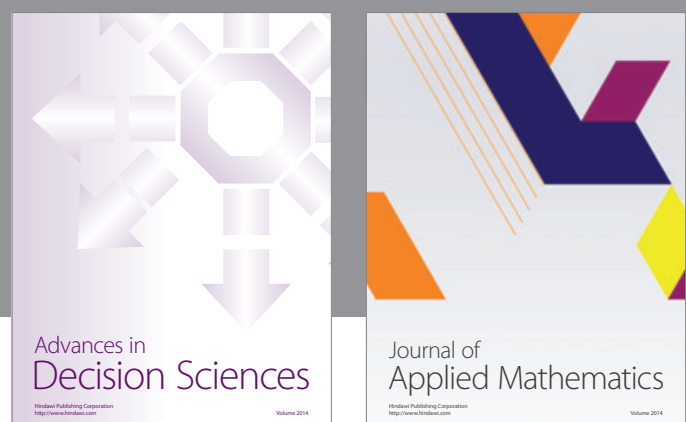

Journal of

Applied Mathematics
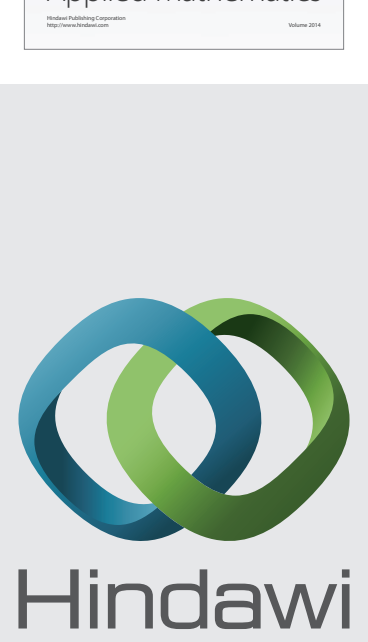

Submit your manuscripts at http://www.hindawi.com
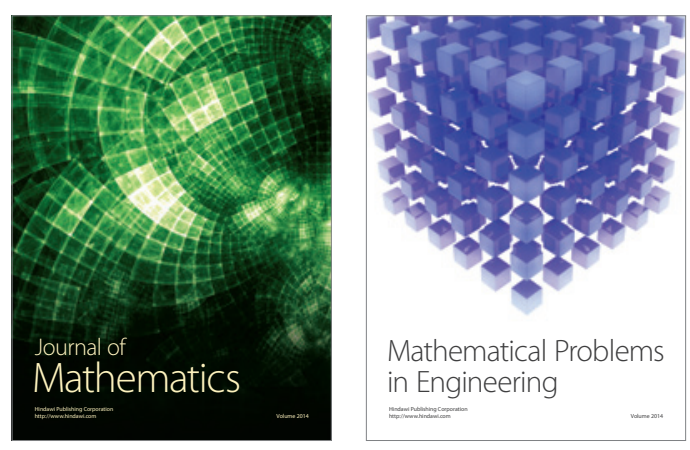

Mathematical Problems in Engineering
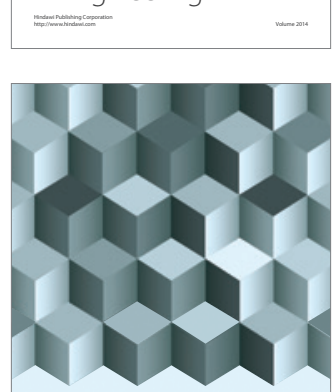

Journal of

Function Spaces
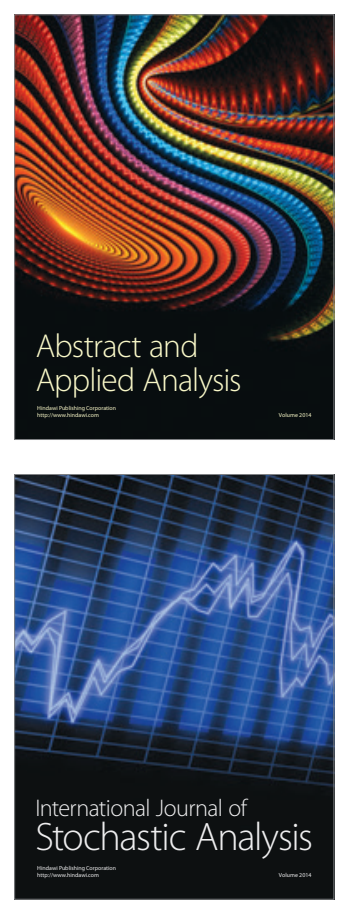

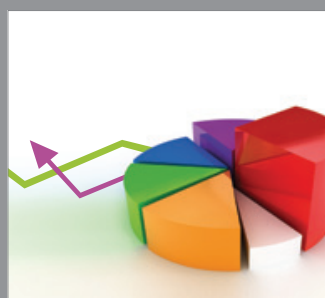

ournal of

Probability and Statistics

Promensencen
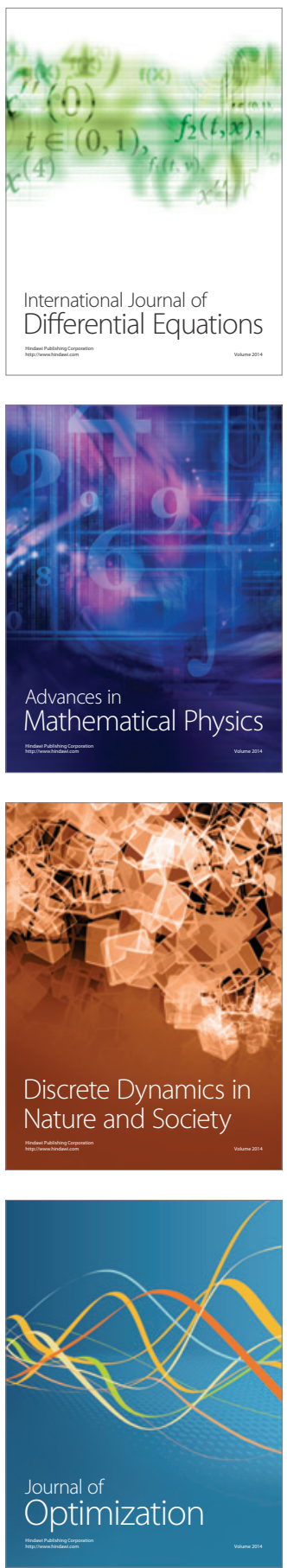